CUBO A Mathematical Journal Vol.15, № 01, (33-47). March 2013

\title{
On Fokker-Planck and linearized Korteweg-de Vries type equations with complex spatial variables 1
}

\author{
Ciprian G. GaL \\ Florida International University, \\ Department of Mathematics, DM 435B, \\ Miami, Florida 33199 \\ cgal@fiu.edu
}

\author{
SORIN G. GAL \\ Department of Mathematics and Computer \\ Science, University of Oradea, \\ Str. Universitatii No. 1 410087, Romania \\ galso@uoradea.ro
}

\begin{abstract}
In the present work, we construct solutions to a Fokker-Planck type equation with real time variable and complex spatial variable, and prove some properties. The equations are obtained from the complexification of the spatial variable by two different methods. Firstly, one complexifies the spatial variable in the corresponding convolution integral in the solution, by replacing the usual sum of variables (translation) by an exponential product (rotation). Secondly, one complexifies the spatial variable directly in the corresponding evolution equation and then one searches for analytic solutions. These methods are also applied to a linear evolution equation related to the Korteweg-de Vries equation.
\end{abstract}

\section{RESUMEN}

En este trabajo construimos soluciones de una ecuación tipo Fokker-Planck con variable de tiempo real y variable espacial compleja. Las ecuaciones se obtienen de la complejización de la variable espacial por dos métodos diferentes. Primero, se complejiza la variable espacial en la integral de convolución respectiva en la solución reemplazando la suma usual de las variables (traslaciones) por un producto de exponenciales (rotación). Luego, se complejiza la variable espacial directamente en la respectiva la ecuación de evolución y se busca por las soluciones analíticas. Estos métodos también se aplican a la ecuación de evolución lineal relacionada a la ecuación Korteweg-de Vries.

Keywords and Phrases: Fokker-Planck equation, Korteweg-de Vries equation, complex convolution integrals, complex spatial variables.

2010 AMS Mathematics Subject Classification: 47D03, 47D06, 47D60.

\footnotetext{
${ }^{1}$ Dedicated to Professor Gaston N'Guerekata on the occasion of his 60th birthday.
} 


\section{Introduction}

Let us consider the following initial value problem:

$$
\left\{\begin{array}{c}
\frac{\partial u}{\partial t}(t, x)=\alpha(t) \frac{\partial^{2} u}{\partial x^{2}}(t, x)+\beta(t) x u(t, x),(t, x) \in \mathbb{R}_{+} \times \mathbb{R} \\
u(0, x)=f(x), x \in \mathbb{R}
\end{array}\right.
$$

where $\alpha \in C\left([0,+\infty), \mathbb{R}_{+}\right)$and $\beta \in C([0,+\infty), \mathbb{R})$. Using the exponential operator method, it is shown in [6] that

$$
u(t, x)=\frac{e^{a(t)+d(t)[c(t) d(t)+b(t)+x]}}{2 \sqrt{\pi c(t)}} \int_{-\infty}^{\infty} e^{-[u+b(t)+2 c(t) d(t)]^{2} /(4 c(t))} f(x-u) d u,
$$

is a solution of (11) provided that the integral in (2) converges, with $a(t), b(t), c(t), d(t)$ depending on $\alpha(t), \beta(t)$, and given in [6, (54)] as follows:

$$
\begin{aligned}
& c(t)=\int_{0}^{t} \alpha(u) d u, d(t)=\int_{0}^{t} \beta(u) d u, \\
& b(t)=-2 \int_{0}^{t}\left[\beta(u) \int_{0}^{u} \alpha(s) d s\right] d u, \\
& a(t)=2 \int_{0}^{t} \beta(u)\left\{\int_{0}^{u}\left[\beta(s) \int_{0}^{s} \alpha(v) d v\right] d s\right\} d u .
\end{aligned}
$$

Note that by the assumptions on $\alpha, \beta$, the functions $a, b, c$ and $d$ are differentiable for every $t>0$, and that $\mathrm{c}(\mathrm{t})>0$, for all $\mathrm{t}>0$. For $\beta(\mathrm{t}) \equiv 0$ and $\alpha(\mathrm{t}) \equiv \mathrm{C}(\mathrm{C}=$ constant $)$, we recapture the initial value problem for the classical heat equation. For $\beta(t) \neq 0, \alpha(t) \neq 0$, the main equation in (11) is known as a Fokker-Planck type equation.

In the second part of this article, we'll devote our attention to the "linearized" Korteweg-de Vries equation with real time variable and complex spatial variable. Indeed, let us consider the well-known Korteweg-de Vries equation

$$
\frac{\partial u}{\partial t}+\alpha u \frac{\partial u}{\partial x}=\frac{\partial^{3} u}{\partial x^{3}}
$$

where $\alpha \in \mathbb{R}$ (see, e.g., Widder [7]), and the related linear problem

$$
\left\{\begin{array}{l}
\frac{\partial u}{\partial t}(t, x)=\frac{\partial^{3} u}{\partial x^{3}}(t, x), \quad(t, x) \in \mathbb{R}_{+} \times \mathbb{R}, \\
\lim _{t \searrow o} u(t, x)=f(x), \quad x \in \mathbb{R} .
\end{array}\right.
$$

For problem (5), the following is known to hold.

Theorem 1.1. ([7, Theorem 4]) Let $f: \mathbb{R} \rightarrow \mathbb{R}$ be continuous and of bounded variation, such that it satisfies the conditions:

(i) The integral

$$
F(s)=\int_{-\infty}^{\infty} e^{-s x} f(x) d x
$$


converges absolutely for $\mathrm{s} \in \mathbb{C}$ with $\operatorname{Re}(\mathrm{s})=\mathrm{c}$;

(ii) Let

$$
\int_{-\infty}^{\infty}|f(c+i \tau)| d \tau<\infty
$$

Setting

$$
u(t, x):=\int_{-\infty}^{\infty} K(x-y, t) f(y) d y=\int_{-\infty}^{\infty} K(u, t) f(x-u) d u
$$

where

$$
\mathrm{K}(\mathrm{u}, \mathrm{t}):=\frac{1}{\pi} \int_{0}^{\infty} \cos \left(\mathrm{u} \tau-\mathrm{t} \tau^{3}\right) \mathrm{d} \tau=\frac{1}{(3 \mathrm{t})^{1 / 3}} \mathrm{Ai}\left(\frac{-\mathrm{u}}{(3 \mathrm{t})^{1 / 3}}\right),
$$

the function $\mathrm{u}(\mathrm{t})$ satisfies (5).

Above,

$$
\operatorname{Ai}(u):=\frac{1}{\pi} \int_{0}^{\infty} \cos \left(u \tau+\tau^{3} / 3\right) d \tau
$$

is also called the Airy function. Moreover, it is well-known (see, e.g., Widder [7, (5.1)]) that

$$
\frac{\partial K}{\partial t}(x, t)=\frac{\partial^{3} K}{\partial x^{3}}(x, t), t>0, x \in \mathbb{R} .
$$

It is natural to ask what happens if in the above equations we complexify the spatial variable and keep the time variable real? We shall proceed as follows. The complexification of the spatial variable in the above mentioned equations is made by two different methods which produce different equations: first, one complexifies the spatial variable in the corresponding formula for the solutions in (2) and (5), respectively, by replacing in the integral the usual sum of variables (translation) by an exponential product(rotation) and looking for solutions in a disk $D_{R}$ of radius $R>1$. This method yields solutions that satisfy differential equations similar to (2) and (5). Secondly, one directly complexifies the spatial variable in the corresponding evolution equations, and then one searches for analytic and non-analytic solutions for the resulting equation. The topic was already developed in detail for complex heat and Laplace equations in [1, 2, for complex wave and telegraph equations in [3] and for complex Schrödinger type equations in [4].

\section{Generalized heat type equations with complex spatial vari- able}

Let $R \geq 1$ and let us now consider the open disk $D_{R}=\{z \in \mathbb{C} ;|z|<R\}$ and $A\left(D_{R}\right)=\left\{f: \bar{D}_{R} \rightarrow \mathbb{C}\right.$; $f$ is analytic on $D_{R}$, continuous on $\left.\bar{D}_{R}\right\}$, endowed with the uniform norm $\|f\|_{R}=\sup \left\{|f(z)| ; z \in \bar{D}_{R}\right\}$. Is well-known that $\left(A\left(D_{R}\right),\|\cdot\|_{R}\right)$ is a Banach space. If $f \in A\left(D_{R}\right)$, then we have $f(z)=\sum_{k=0}^{\infty} a_{k} z^{k}$, for all $z \in D_{R}$. Finally, $\omega_{1}(f ; \delta)_{\bar{D}_{R}}$ denotes the modulus of continuity,

$$
\omega_{1}(f ; \delta)_{D_{R}}=\sup \left\{|f(u)-f(v)| ;|u-v| \leq \delta, u, v \in \bar{D}_{R}\right\} .
$$


Concerning the system (1), we will first complexify the solution in (2) as follows. For $f \in A\left(D_{R}\right)$ and $t>0$, let us replace $x$ and the translation $x-u$ in (2) by $z$ and the rotation $z e^{-i u}$, respectively, and consider the complex integral

$$
G_{t}(f)(z)=\frac{e^{a(t)+d(t)[c(t) d(t)+b(t)+z]}}{2 \sqrt{\pi c(t)}} \int_{-\infty}^{\infty} e^{-[u+b(t)+2 c(t) d(t)]^{2} /(4 c(t))} f\left(z e^{-i u}\right) d u, \quad z \in \bar{D}_{R} .
$$

The first goal of this section is to prove some properties of the complex integral (9).

Theorem 2.1. Let $\mathrm{R}>1$ and $\mathrm{f} \in \mathrm{A}\left(\mathrm{D}_{\mathrm{R}}\right)$.

(i) For all $t>0, G_{t}(f) \in A\left(D_{R}\right)$, namely $G_{t}(f)$ is continuous on $\bar{D}_{R}$, is analytic in $D_{R}$, and the following holds:

$$
G_{t}(f)(z)=e^{\Phi(t, z)} \sum_{k=0}^{\infty} a_{k} d_{k}(t) z^{k}, z \in D_{R}
$$

where

$$
\Phi(t, z)=a(t)+d(t)[c(t) d(t)+b(t)+z]
$$

and, for all $\mathrm{k} \geq 0$,

$$
\mathrm{d}_{\mathrm{k}}(\mathrm{t}):=\mathrm{e}^{-\mathrm{k}^{2} \mathrm{c}(\mathrm{t})+\mathrm{ikg}(\mathrm{t})}, g(t):=b(t)+2 c(t) d(t) .
$$

(ii) Setting

$$
W_{t}(f)(z):=e^{-\Phi(t, z)} G_{t}(f)(z)=\frac{1}{2 \sqrt{\pi c(t)}} \int_{-\infty}^{\infty} f\left(z e^{-i \mathfrak{u}}\right) e^{-[u+g(t)]^{2} /(4 c(t))} d u
$$

the following estimate holds:

$$
\left|W_{t}(f)(z)-f(z)\right| \leq(R+1)\left[1+\frac{2}{\sqrt{\pi}}+\frac{|g(t)|}{2 \sqrt{c(t)}}\right] \omega_{1}(f ; \sqrt{c(t)}) \bar{D}_{R},
$$

for all $z \in \overline{\mathrm{D}}_{\mathrm{R}}, \mathrm{t}>0$.

(iii) The operator $W_{\mathrm{t}}$ is contractive, that is, $\left\|W_{t}(f)\right\|_{\bar{D}_{R}} \leq\|f\|_{\bar{D}_{R}}$, for all $t>0, f \in A\left(D_{R}\right)$.

(iv) Let

$$
\mathrm{U}(\mathrm{t}, \varphi):=e^{\mathrm{a}(\mathrm{t}) \mathrm{d}(\mathrm{t})[\mathrm{c}(\mathrm{t}) \mathrm{d}(\mathrm{t})+\mathrm{b}(\mathrm{t})+\varphi]} \sum_{k=0}^{\infty} \mathrm{a}_{k} \mathrm{~d}_{\mathrm{k}}(\mathrm{t}) z^{\mathrm{k}},
$$

for every $z \neq 0, z=r e^{-i \varphi}$ such that $\mathrm{r} \in(0, \mathrm{R}), \varphi \in(0,2 \pi]$. Then, $\mathrm{U}(\mathrm{t}, \varphi)$ satisfies

$$
\frac{\partial \mathrm{U}}{\partial \mathrm{t}}(\mathrm{t}, \varphi)=\alpha(\mathrm{t}) \frac{\partial^{2} \mathrm{U}}{\partial \varphi^{2}}(\mathrm{t}, \varphi)+\beta(\mathrm{t}) \varphi \mathrm{U}(\mathrm{t}, \varphi)
$$

for $(\mathrm{t}, z) \in \mathbb{R}_{+} \times \mathrm{D}_{\mathrm{R}} \backslash\{0\}, z=\mathrm{r} e^{i \varphi}, \mathrm{r} \in(0, \mathrm{R})$, and

$$
\mathrm{U}(0, \varphi)=\mathrm{f}\left(\mathrm{r} e^{i \varphi}\right), \varphi \in(0,2 \pi], 0<\mathrm{r} \leq \mathrm{R}, \mathrm{f} \in \mathrm{A}\left(\mathrm{D}_{\mathrm{R}}\right) .
$$


Proof. (i) For fixed $z \in D_{R}$, we have

$$
f\left(z e^{-i u}\right)=\sum_{k=0}^{\infty} a_{k} e^{-i k u} z^{k}
$$

Since $\left|a_{k} e^{-i k u}\right|=\left|a_{k}\right|$, for all $u \in \mathbb{R}$, and since $\sum_{k=0}^{\infty} a_{k} z^{k}$ is absolutely convergent, it follows that $\sum_{k=0}^{\infty} a_{k} e^{-i k u} z^{k}$ is uniformly convergent with respect to $u \in \mathbb{R}$. Thus, on account of (13), we can integrate in (9) term by term. This yields

$$
\begin{aligned}
\mathrm{G}_{\mathrm{t}}(\mathrm{f})(z) & =e^{\Phi(z)} \sum_{k=0}^{\infty} a_{k}\left[\frac{1}{2 \sqrt{\pi \mathrm{c}(\mathrm{t})}} \int_{-\infty}^{+\infty} e^{-i k u} \cdot e^{-[u+g(t)]^{2} /(4 \mathrm{c}(\mathrm{t}))} \mathrm{du}\right] z^{\mathrm{k}} \\
& ==e^{\Phi(z)} \sum_{k=0}^{\infty} a_{k}\left[\frac { 1 } { 2 \sqrt { \pi c ( t ) } } \int _ { - \infty } ^ { + \infty } \left(\cos \left[k(v-g(t)]-i \sin [k(v-g(t)]) e^{-v^{2} /(4 c(t))} d v\right] z^{k}\right.\right. \\
& :=e^{\Phi(z)}\left[\mathrm{I}_{1}-i \mathrm{I}_{2}\right] .
\end{aligned}
$$

Since $\sin (k v) e^{-v^{2}} /(4 c(t))$ is odd as function of $v$, we have

$$
\begin{aligned}
\mathrm{I}_{1} & =\sum_{k=0}^{\infty} a_{k}\left[\cos (k g(t)) \frac{1}{2 \sqrt{\pi c(t)}} \int_{-\infty}^{+\infty} \cos (k v) e^{-v^{2} /(4 c(t))} d v\right] z^{k} \\
& =\sum_{k=0}^{\infty} a_{k}\left[\cos (k g(t)) \cdot e^{-k^{2} c(t)}\right] z^{k}
\end{aligned}
$$

and

$$
\mathrm{I}_{2}=-\sum_{\mathrm{k}=0}^{\infty} \mathrm{a}_{\mathrm{k}}\left[\sin (\mathrm{kg}(\mathrm{t})) \mathrm{e}^{-\mathrm{k}^{2} \mathrm{c}(\mathrm{t})}\right] z^{\mathrm{k}} .
$$

Hence, these calculations give the formula (10) and prove the analyticity of $G_{t}(f)(z)$, as function of $z \in \mathrm{D}_{\mathrm{R}}$. To prove the continuity in $\overline{\mathrm{D}}_{\mathrm{R}}$, it suffices to prove the continuity in $z$, of the function

$$
H_{t}(f)(z):=\frac{1}{2 \sqrt{\pi c(t)}} \int_{-\infty}^{\infty} e^{-[u+g(t)]^{2} /(4 \mathfrak{c}(t))} f\left(z e^{-i \mathfrak{u}}\right) d u .
$$

To this end, let $z_{0}, z_{n} \in \bar{D}_{R}$ be such that $\lim _{n \rightarrow \infty} z_{n}=z_{0}$. We get

$$
\begin{aligned}
& \left|H_{\mathfrak{t}}(f)\left(z_{\mathfrak{n}}\right)-H_{t}(f)\left(z_{0}\right)\right| \leq \frac{1}{2 \sqrt{\pi c(t)}} \int_{-\infty}^{+\infty}\left|f\left(z_{\mathfrak{n}} e^{-i \mathfrak{u}}\right)-f\left(z_{0} e^{-i \mathfrak{u}}\right)\right| e^{-[\mathfrak{u}+g(t)]^{2} /(4 \mathfrak{c}(\mathrm{t}))} d \mathfrak{u} \\
& \leq \frac{1}{2 \sqrt{\pi \mathrm{c}(\mathrm{t})}} \int_{-\infty}^{+\infty} \omega_{1}\left(f ;\left|z_{\mathrm{n}} e^{-i \mathrm{u}}-z_{0} e^{-i \mathfrak{u}}\right|\right)_{\bar{D}_{R}} e^{-[\mathrm{u}+\mathrm{g}(\mathrm{t})]^{2} /(4 \mathrm{c}(\mathrm{t}))} \mathrm{du} \\
& =\frac{1}{2 \sqrt{\pi \mathrm{c}(\mathrm{t})}} \int_{-\infty}^{+\infty} \omega_{1}\left(f ;\left|z_{\mathrm{n}}-z_{0}\right|\right)_{\bar{D}_{\mathrm{R}}} e^{-[u+g(t)]^{2} /(4 \mathrm{c}(\mathrm{t}))} \mathrm{du} \\
& =\omega_{1}\left(f ;\left|z_{\mathfrak{n}}-z_{0}\right|\right)_{\bar{D}_{\mathrm{R}}} \text {. }
\end{aligned}
$$


Passing to the limit as $n \rightarrow \infty$, the continuity of $H_{t}(f)(z)$ at $z_{0} \in \bar{D}_{R}$ is a consequence of (14) since $f$ is continuous on $\bar{D}_{R}$.

(ii) First, note that we can also write

$$
W_{t}(f)(z)=\frac{1}{2 \sqrt{\pi c(t)}} \int_{-\infty}^{+\infty} e^{-v^{2} /(4 c(t))} f\left(z e^{-i(v-g(t))}\right) d v .
$$

A simple calculation gives

$$
\begin{aligned}
\left|W_{t}(f)(z)-f(z)\right| & \leq \frac{1}{2 \sqrt{\pi c}} \int_{-\infty}^{+\infty}\left|f\left(z e^{-i(u-g)}\right)-f(z)\right| e^{-u^{2} /(4 c)} d u \\
& \leq \frac{R+1}{2 \sqrt{\pi c}} \int_{-\infty}^{\infty} \omega_{1}\left(f ;\left|1-e^{-i(u-g)}\right|\right)_{\bar{D}_{R}} e^{-u^{2} /(4 c)} d u \\
& =\frac{R+1}{2 \sqrt{\pi T}} \int_{-\infty}^{+\infty} \omega_{1}\left(f ; 2\left|\sin \frac{u-g}{2}\right|\right)_{\bar{D}_{R}} e^{-u^{2} /(4 c)} d u \\
& \leq \frac{R+1}{2 \sqrt{\pi T}} \int_{-\infty}^{+\infty} \omega_{1}(f ;|u-g|)_{\bar{D}_{R}} e^{-u^{2} /(4 c)} d u \\
& \leq \frac{R+1}{2 \sqrt{\pi T}} \int_{-\infty}^{+\infty} \omega_{1}(f ; \sqrt{c})_{\bar{D}_{R}}\left(\frac{|u-g|}{\sqrt{c(t)}}+1\right) e^{-u^{2} /(4 c)} d u \\
& \leq(R+1)\left[\omega_{1}(f ; \sqrt{c})_{\bar{D}_{R}}+\frac{\omega_{1}(f ; \sqrt{c})_{\bar{D}_{R}}}{\sqrt{c} 2 \sqrt{\pi c}} \int_{0}^{\infty} 2 u e^{-u^{2} /(4 c)} d u\right] \\
& +(R+1)\left[|A| \cdot \frac{\omega_{1}(f ; \sqrt{c})_{\bar{D}_{R}}}{\sqrt{c} 2 \sqrt{\pi c}} \int_{0}^{\infty} e^{-u^{2} /(4 c)} d u\right] .
\end{aligned}
$$

Since $\int_{0}^{\infty} 2 u e^{-u^{2} /(4 c(t))} d u=4 c(t)$, we infer

$$
\left|W_{t}(f)(z)-f(z)\right| \leq \omega_{1}(f ; \sqrt{c})_{\bar{D}_{R}}\left[1+\frac{2}{\sqrt{\pi}}+\frac{|g|}{2 \sqrt{c}}\right](R+1) .
$$

This proves (ii).

(iii) Since $\frac{1}{2 \sqrt{\pi c}} \int_{-\infty}^{+\infty} e^{-u^{2} /(4 c)} d u=1$, we also have

$$
\left|W_{t}(f)(z)\right| \leq \frac{1}{2 \sqrt{\pi c}} \int_{-\infty}^{+\infty}\left|f\left(z e^{-i(u-g)}\right)\right| e^{-u^{2} /(4 \mathfrak{c})} d u \leq\|f\|_{\bar{D}_{R}}, \quad z \in \bar{D}_{R},
$$

which proves the claim.

(iv) Let $f \in A\left(D_{R}\right)$, and $z \in D_{R}, z=r e^{i \varphi}, 0<r<R$. Set

$$
B(t, \varphi):=a(t)+d(t)[c(t) d(t)+b(t)+\varphi], A_{k}(t):=-k^{2} c(t)+i k g(t) ;
$$

by (i), we have $d_{k}(t)=e^{A_{k}(t)}$, and we can write

$$
\mathrm{U}(\mathrm{t}, \varphi)=e^{\mathrm{B}(\mathrm{t}, \varphi)} \sum_{k=0}^{\infty} a_{k} e^{A_{k}(t)} r^{k} e^{k i \varphi} .
$$


Consequently, since the series representation (10) for $G_{t}(f)(z)$ is uniformly convergent in any compact disk included in $\mathrm{D}_{\mathrm{R}}$, it follows that $\mathrm{U}(\mathrm{t}, \varphi)$ can be differentiated term by term with respect to $t$ and $\varphi$. Therefore, simple calculations show that $\mathrm{U}$, given by (16), satisfies. We emphasize again that in (11) we must take $z \neq 0$ simply because $z=0$ has no polar representation, that is, $z=0$ cannot be represented as function of $\varphi$. Finally, it is also easy to check that $\mathrm{U}(\mathrm{t}, \varphi)$ satisfies (12) since $a(0)=b(0)=c(0)=d(0)=0$ on account of (3). This completes the proof of the theorem.

Remark 2.2. In Theorem 2.1-(ii), we have $\lim _{\mathrm{t} \rightarrow 0} \mathrm{c}(\mathrm{t})=0$. Therefore, there exists a sufficiently small $\delta_{0}>0$ such that $0<\mathrm{c}(\mathrm{t})<1$, for all $\mathrm{t} \in\left[0, \delta_{0}\right)$. This implies that

$$
|g(t)| / \sqrt{c(t)} \leq|b(t)| / c(t)+2|d(t)|
$$

for all $\mathrm{t} \in\left(0, \delta_{0}\right)$. Exploiting (3) once more again it is easy to show that $|\mathrm{b}(\mathrm{t})| / \mathrm{c}(\mathrm{t}) \leq 2 \beta_{0} \mathrm{t}$, for all $\mathrm{t} \in\left(0, \delta_{0}\right)$, where $\beta_{0}=\|\beta\|_{\left[0, \delta_{0}\right]}$. This together with the inequality [17) yields $\lim _{\mathrm{t} \rightarrow 0}|\mathrm{~g}(\mathrm{t})| / \sqrt{\mathrm{c}(\mathrm{t})}=$ 0 . As a consequence, $c f$. the estimate of Theorem 2.1-(ii), it also follows that $\lim _{\mathrm{t} \backslash \mathrm{0}} \mathrm{W}_{\mathrm{t}}(\mathrm{f})(z)=$ $\mathrm{f}(z)$, for all $z \in \overline{\mathrm{D}}_{\mathrm{R}}$.

In what follows, the system (11) is complexified, by replacing $x \in \mathbb{R}$ with $z \in \mathbb{C}$ directly in the equations. More precisely, our goal is to study the following initial value problem

$$
\left\{\begin{array}{c}
\frac{\partial u}{\partial t}(t, z)=\alpha(t) \frac{\partial^{2} u}{\partial z^{2}}(t, z)+\beta(t) z u(t, z) \\
u(0, z)=f(z)
\end{array}\right.
$$

We will first consider the case when $f$ is analytic. Our first goal is to search for analytic solutions $\mathfrak{u}(\mathrm{t}, z)$, as functions of $z$, for any $\mathrm{t}>0$. First, we need some basic notations. For $r>0$, define the strip

$$
S_{r}=\{z=x+i y \in \mathbb{C} ; x \in \mathbb{R},|y| \leq r\}
$$

and

$$
A\left(S_{r}\right)=\left\{f: S_{r} \rightarrow C ; f \text { is analytic in } S_{r}\right\},
$$

(i.e., $f$ is analytic in a domain that contains $\left.S_{r}\right)$. Next, let $\mathcal{M}_{r}$ be the set of all $f \in A\left(S_{r}\right)$ such that there exists $g \in \mathrm{L}^{1}\left(\mathbb{R}_{+}\right) \cap \mathrm{L}^{\infty}\left(\mathbb{R}_{+}\right)$with the property that $\left|\mathrm{f}^{\prime}(z)\right| \leq \mathrm{g}(|z|)$, as $|z|$ goes to infinity. Finally, let

$$
u(t, z):=\frac{e^{\Phi(t, z)}}{2 \sqrt{\pi c(t)}} \int_{-\infty}^{\infty} f(z-\xi) e^{-[\xi+g(t)]^{2} /(4 c(t))} d \xi,(t, z) \in \mathbb{R}_{+} \times S_{r},
$$

where $c(t), \Phi(t, z)$, and $g(t)$ are defined in the statement of Theorem 2.1.(i).

Theorem 2.3. For each $\mathrm{f} \in \mathcal{M}_{\mathrm{r}}$ we have $\mathrm{u}=\mathfrak{u}(\mathrm{t}, \cdot) \in \mathrm{A}\left(\mathrm{S}_{\mathrm{r}}\right)$, for any $\mathrm{t}>0$. Moreover, $\mathfrak{u}(\mathrm{t}, z)$ solves (18) for $(\mathrm{t}, z) \in \mathbb{R}_{+} \times \mathrm{S}_{\mathrm{r}}$. 
Proof. If $\mathrm{f} \in \mathcal{M}_{\mathrm{r}}$, there exists $\mathrm{M}>0$ such that

$$
\sup \left\{\left|f^{\prime}(z)\right| ; z \in S_{r}\right\}=\left\|f^{\prime}\right\|_{S_{r}} \leq M .
$$

By the Mean Value Theorem in complex analysis, $f$ is uniformly continuous in $S_{r}$, and that

$$
|f(z-\xi)| \leq|f(0)|+\left\|f^{\prime}\right\|_{S_{r}} \cdot|z-\xi| \leq|f(0)|+M|z-\xi| .
$$

The latter implies that the integral $I(t, z):=\frac{1}{2 \sqrt{\pi c(t)}} \int_{-\infty}^{\infty} f(z-\xi) e^{-[\xi+g(t)]^{2} /(4 c(t))} d \xi$ exists and is absolutely convergent in $\mathbb{C}$, and that $\mathrm{I}(\mathrm{t}, z)$ is differentiable with respect to any $z \in S_{\mathrm{r}}$, with

$$
\partial_{z} I(t, z)=\int_{-\infty}^{\infty} f^{\prime}(z-\xi) e^{-[\xi+g(t)]^{2} /(4 c(t))} d \xi .
$$

The analicity of $\Phi(t, z)$ with respect to $z \in S_{r}$ implies that $u=u(t, \cdot)$ also belongs to $A\left(S_{r}\right)$, for any $t>0$. Analogous calculations to those performed in (15) yield

$$
\begin{aligned}
& |I(t, z)-f(z)| \\
& \leq \omega_{1}(f ;|g(t)|)_{S_{r}}+\omega_{1}(f ; \sqrt{c(t)})_{S_{r}} \frac{1}{2 \sqrt{\pi c(t)}} \int_{-\infty}^{\infty}\left(|u|(c(t))^{-1 / 2}+1\right) e^{-u^{2} /(4 c(t))} d u \\
& =\omega_{1}(f ;|g(t)|)_{S_{r}}+\left(1+\frac{2}{\sqrt{\pi}}\right) \omega_{1}(f ; \sqrt{c(t)}) s_{r} .
\end{aligned}
$$

Taking now into account the Remark 2.2, and the uniform continuity of $f$ on $S_{r}$, it easily follows that $\lim _{t \searrow 0} I(t, z)=f(z)$, for all $z \in S_{r}$. This together with the fact that $\Phi(0, z)=1$ yields $u(0, z)=\lim _{t \searrow o} u(t, z)=f(z)$, for all $z \in S_{r}$, i.e., $u$ satisfies the initial condition of (18). It remains to show that $u$ also solves the main equation of (18). To this end, define

$$
F(t, z):=\frac{\partial u}{\partial t}(t, z)-\alpha(t) \frac{\partial^{2} u}{\partial z^{2}}(t, z)-\beta(t) z u(t, z)
$$

for all $(t, z) \in \mathbb{R}_{+} \times S_{r}$, and recall that $u(0, z)=f(z), z \in S_{r}$. For each $t>0, F(t, \cdot)$ is analytic in $S_{\mathrm{r}}$. Taking now $z=x \in \mathbb{R}$ in all the equations of (18), we can now apply known theory to deduce that $u(t, z)=u(t, x)$ also solves (1). Hence, $F(t, x)=0$, for all $(t, x) \in \mathbb{R}_{+} \times \mathbb{R}$. The identity theorem for holomorphic functions (in a domain that contains $S_{r}$ ) implies that we must also have $\mathrm{F}(\mathrm{t}, z)=0,(\mathrm{t}, z) \in \mathbb{R}_{+} \times \mathrm{S}_{\mathrm{r}}$. This finishes the proof of the theorem.

\section{$3 \quad$ Linearized Korteweg-de Vries type equations}

For $\mathrm{R}>1$, let us define the open disk

$$
\mathrm{D}_{\mathrm{R}}:=\{z \in \mathbb{C}:|z|<\mathrm{R}\} .
$$

Next we endow the local convex space

$$
A^{*}\left(D_{R}\right):=\left\{f: \bar{D}_{R} \rightarrow \mathbb{C}: f \text { is analytic on } D_{R}\right\}
$$


with the countable family of seminorms

$$
\|\mathbf{f}\|_{\mathrm{n}}:=\sup \left\{|\mathrm{f}(z)|: z \in \overline{\mathrm{D}}_{\mathrm{R}_{\mathrm{n}}}\right\}, \mathrm{R}_{\mathrm{n}} \nearrow \mathrm{R}, \mathrm{R}_{\mathrm{n}} \geq 1,
$$

and metric

$$
d(f, g):=\sum_{n=0}^{\infty} \frac{1}{2^{n}} \frac{\|f-g\|_{n}}{1+\|f-g\|_{n}} .
$$

Then $A^{*}\left(D_{R}\right)$ is a Fréchet space.

Let $f \in A^{*}\left(D_{R}\right)$ such that

$$
f(z)=\sum_{k=0}^{\infty} a_{k} z^{k}
$$

for all $z \in \mathrm{D}_{\mathrm{R}}$. We consider the integral operator

$$
\mathcal{T}_{\mathrm{K}}(\mathrm{t})(\mathrm{f})(z):=\int_{-\infty}^{\infty} \mathrm{K}(\mathrm{u}, \mathrm{t}) \mathrm{f}\left(z e^{-i \mathrm{u}}\right) \mathrm{d} \mathrm{u}, z \in \mathrm{D}_{\mathrm{R}},
$$

with $\mathrm{K}(\mathrm{u}, \mathrm{t})$ given by $(7)$. Evidently, since $\mathrm{K}(-\mathfrak{u}, \mathrm{t}) \neq \mathrm{K}(\mathrm{u}, \mathrm{t})$, we can naturally introduce another complex integral by

$$
\widetilde{\mathcal{T}}_{\mathrm{K}}(\mathrm{t})(\mathrm{f})(z):=\int_{-\infty}^{\infty} \mathrm{K}(-\mathrm{u}, \mathrm{t}) \mathrm{f}\left(z \mathrm{e}^{\mathrm{iu}}\right) \mathrm{du}, z \in \mathrm{D}_{\mathrm{R}} .
$$

The first goal of this section is to prove some properties for (19) and (20).

Theorem 3.1. Let $\mathrm{R}>1, \mathrm{f} \in \mathrm{A}^{*}\left(\mathrm{D}_{\mathrm{R}}\right)$, that is, $\mathrm{f}(z)=\sum_{\mathrm{k}=0}^{\infty} \mathrm{a}_{\mathrm{k}} z^{\mathrm{k}}$, for all $z \in \mathrm{D}_{\mathrm{R}}$. Let $\mathcal{T}_{\mathrm{K}}(\cdot)(\mathbf{f})$ and $\widetilde{\mathcal{T}}_{\mathrm{K}}(\cdot)(\mathrm{f})$ be as in (19) and (20), respectively.

(i) For all $\mathrm{t} \geq 0$, as functions of $z$, we have $\mathcal{T}_{\mathrm{K}}(\cdot)(\mathrm{f})(z) \in \mathrm{A}^{*}\left(\mathrm{D}_{\mathrm{R}}\right), \widetilde{\mathrm{T}}_{\mathrm{K}}(\cdot)(\mathrm{f})(z) \in \mathrm{A}^{*}\left(\mathrm{D}_{\mathrm{R}}\right)$, and there hold

$$
\mathcal{T}_{K}(t)(f)(z)=\sum_{k=0}^{\infty} a_{k} d_{k}(t) z^{k} \quad \text { and } \quad \widetilde{\mathcal{T}}_{K}(t)(f)(z)=\sum_{k=0}^{\infty} a_{k} b_{k}(t) z^{k}, z \in D_{R},
$$

where for all $\mathrm{k} \geq 0$,

$$
d_{k}(t)=e^{i t k^{3}} \text { and } \quad b_{k}(t)=e^{-i t k^{3}}
$$

Moreover,

$$
\mathcal{T}_{\mathrm{K}}(0)(f)=\widetilde{\mathcal{T}}_{\mathrm{K}}(0)(\mathrm{f})=\mathrm{f} .
$$

(ii) For all $z \in \mathrm{D}_{\mathrm{r}}$ with $1 \leq \mathrm{r}<\mathrm{R}$ and $\mathrm{t} \in \mathbb{R}_{+}$, the following estimate holds:

$$
\left|\mathcal{T}_{K}(t)(f)(z)-f(z)\right| \leq \frac{t^{2}}{2} \sum_{k=0}^{\infty}\left|a_{k}\right| k^{6} r^{k}+|t| \sum_{k=0}^{\infty}\left|a_{k}\right| k^{3} r^{k},
$$

where

$$
\sum_{k=0}^{\infty}\left|a_{k}\right| k^{6} r^{k}<\infty, \text { since } f^{(6)} \in A^{*}\left(D_{R}\right)
$$


(iii) For all $z \in \mathrm{D}_{\mathrm{r}}$ with $1 \leq \mathrm{r}<\mathrm{R}$ and $\mathrm{t}, \mathrm{s} \in \mathbb{R}_{+}$, there holds:

$$
\left|\mathcal{T}_{K}(t)(f)(z)-\mathcal{T}_{K}(s)(f)(z)\right| \leq 2 \sum_{k=0}^{\infty}\left|a_{k}\right| k^{3} r^{k}|t-s|
$$

and

$$
\left|\widetilde{\mathcal{T}}_{\mathrm{K}}(\mathrm{t})(\mathrm{f})(z)-\widetilde{\mathcal{T}}_{\mathrm{K}}(\mathrm{s})(\mathrm{f})(z)\right| \leq 2 \sum_{\mathrm{k}=0}^{\infty}\left|\mathrm{a}_{\mathrm{k}}\right| \mathrm{k}^{3} \mathrm{r}^{\mathrm{k}}|\mathrm{t}-\mathrm{s}|
$$

(iv) The families $\left\{\mathcal{T}_{\mathrm{K}}(\mathrm{t})\right\}_{\mathrm{t} \geq 0}$ and $\left\{\widetilde{\mathcal{T}}_{\mathrm{K}}(\mathrm{t})\right\}_{\mathrm{t}>0}$ are $\left(\mathrm{C}_{0}\right)$-semigroups of linear operators, locally equicontinuous (that is, equicontinuous for $\mathrm{t} \in[0, \mathrm{a}]$, for some $\mathrm{a}>0$ ) on $\mathrm{A}^{*}\left(\mathrm{D}_{\mathrm{R}}\right)$. For each $\mathrm{f} \in \mathrm{A}^{*}\left(\mathrm{D}_{\mathrm{R}}\right)$, the corresponding Cauchy problems

$$
\left\{\begin{array}{lc}
\frac{\partial u}{\partial t}+\frac{\partial^{3} u}{\partial \varphi^{3}}=0, & (t, z) \in \mathbb{R}_{+} \times D_{R} \backslash\{0\} \\
u(0, z)=f(z), & z \in \bar{D}_{R}
\end{array}\right.
$$

and

$$
\left\{\begin{array}{cc}
\frac{\partial w}{\partial t}=\frac{\partial^{3} w}{\partial \varphi^{3}}, & (t, z) \in \mathbb{R}_{+} \times D_{R} \backslash\{0\} \\
u(0, z)=f(z), & z \in \bar{D}_{R}
\end{array}\right.
$$

are well-posed, with solutions given by

$$
\begin{aligned}
& \mathrm{u}(\mathrm{t})=\mathcal{T}_{\mathrm{K}}(\mathrm{t})(\mathrm{f}) \in \mathrm{C}^{\infty}\left(\mathbb{R}_{+} ; A^{*}\left(\mathrm{D}_{\mathrm{R}}\right)\right), \\
& w(\mathrm{t})=\widetilde{\mathcal{T}}_{\mathrm{K}}(\mathrm{t})(\mathrm{f}) \in \mathrm{C}^{\infty}\left(\mathbb{R}_{+} ; A^{*}\left(\mathrm{D}_{\mathrm{R}}\right)\right),
\end{aligned}
$$

respectively.

Proof. We will prove the above statements only for the family $\left\{\mathcal{T}_{K}(t)\right\}_{t \geq 0}$ (the proof for $\left\{\widetilde{\mathcal{T}}_{K}(t)\right\}_{t \geq 0}$ is the same).

(i) Let $f(z)=\sum_{k=0}^{\infty} a_{k} z^{k}$, for all $z \in D_{R}$. For fixed $z \in D_{R}$, we get

$$
f\left(z e^{-i u}\right)=\sum_{k=0}^{\infty} a_{k} e^{-i k u} z^{k}
$$

Since $\left|a_{k} e^{-i k u}\right|=\left|a_{k}\right|$, for all $u \in \mathbb{R}$, and since $\sum_{k=0}^{\infty} a_{k} z^{k}$ is absolutely convergent, the series $\sum_{k=0}^{\infty} a_{k} e^{-i k u} z^{k}$ is also uniformly convergent with respect to $u \in \mathbb{R}$. Therefore, the latter can be integrated term by term. Using (19), we deduce

$$
\begin{aligned}
\mathcal{T}_{K}(t)(f)(z) & =\sum_{k=0}^{\infty} a_{k}\left\{\int_{-\infty}^{\infty}\left[\frac{1}{\pi} \int_{0}^{+\infty} \cos \left(u \tau-t \tau^{3}\right) d \tau\right] e^{-i k u} d u\right\} z^{k} \\
& =\sum_{k=0}^{\infty} a_{k} d_{k}(t) z^{k}
\end{aligned}
$$


where

$$
\begin{aligned}
d_{k}(t) & =\int_{-\infty}^{\infty}\left[\frac{1}{\pi} \int_{0}^{+\infty} \cos \left(u \tau-t \tau^{3}\right) d \tau\right] \cos (k u) d u \\
& -i \int_{-\infty}^{\infty}\left[\frac{1}{\pi} \int_{0}^{+\infty} \cos \left(u \tau-t \tau^{3}\right) d \tau\right] \sin (k u) d u \\
& =\int_{-\infty}^{0}\left[\frac{1}{\pi} \int_{0}^{+\infty} \cos \left(u \tau-t \tau^{3}\right) d \tau\right] \cos (k u) d u \\
& +\int_{0}^{\infty}\left[\frac{1}{\pi} \int_{0}^{+\infty} \cos \left(u \tau-t \tau^{3}\right) d \tau\right] \cos (k u) d u \\
& -i \int_{-\infty}^{0}\left[\frac{1}{\pi} \int_{0}^{+\infty} \cos \left(u \tau-t \tau^{3}\right) d \tau\right] \sin (k u) d u \\
& +\int_{0}^{\infty}\left[\frac{1}{\pi} \int_{0}^{+\infty} \cos \left(u \tau-t \tau^{3}\right) d \tau\right] \sin (k u) d u \\
& =\frac{1}{\pi} \int_{0}^{\infty}\left[\int_{0}^{\infty}\left(\cos \left(u \tau+t \tau^{3}\right)+\cos \left(u \tau-t \tau^{3}\right)\right) d \tau\right] \cos (k u) d u \\
& +\frac{i}{\pi}\left\{\int_{0}^{\infty}\left[\int_{0}^{\infty}\left(\cos \left(u \tau+t \tau^{3}\right)-\cos \left(u \tau-t \tau^{3}\right)\right) d \tau\right] \sin (k u) d u\right\} .
\end{aligned}
$$

On the other hand, it is well-known that the Fourier transform of the Airy's function is given by (see, e.g., [5, p. 87, Table 4.2])

$$
\int_{-\infty}^{\infty}\left[\frac{1}{\pi} \int_{0}^{\infty} \cos \left(u \tau+\tau^{3} / 3\right) d \tau\right] e^{-i k \omega u} d u=e^{i(\omega k)^{3} / 3}=: I_{1}+I_{2}
$$

where

$$
\begin{aligned}
& I_{1}:=\frac{1}{\pi} \int_{0}^{\infty}\left[\int_{0}^{\infty}\left(\cos \left(\tau^{3} / 3+u \tau\right)+\cos \left(\tau^{3} / 3-u \tau\right)\right) d \tau\right] \cos (k \omega u) d u, \\
& I_{2}:=\frac{i}{\pi}\left\{\int_{0}^{\infty}\left[\int_{0}^{\infty}\left(\cos \left(\tau^{3} / 3+u \tau\right)-\cos \left(\tau^{3} / 3-u \tau\right)\right) d \tau\right] \sin (k \omega u) d u\right\} .
\end{aligned}
$$

Now, by a change of variable $\tau=(3 t)^{1 / 3} \eta$, and then another $u=\frac{v}{(3 t)^{1 / 3}},(t>0$ is a fixed parameter), simple calculations yield

$$
\begin{aligned}
\mathrm{I}_{1}+\mathrm{I}_{2} & =\frac{1}{\pi} \int_{0}^{\infty}\left[\int_{0}^{\infty}\left(\cos \left(\mathrm{t}^{3}+v \eta\right)+\cos \left(\mathrm{t}^{3}-v \eta\right)\right) \mathrm{d} \eta\right] \cos \left(k \omega v /(3 \mathrm{t})^{1 / 3}\right) \mathrm{d} v \\
& +\frac{i}{\pi} \int_{0}^{\infty}\left[\int_{0}^{\infty}\left(\cos \left(\mathrm{t}^{3}+v \eta\right)-\cos \left(\mathrm{t}^{3}-v \eta\right)\right) \mathrm{d} \eta\right] \sin \left(k \omega v /(3 \mathrm{t})^{1 / 3}\right) \mathrm{d} \nu \\
& =e^{i(\omega k)^{3} / 3} .
\end{aligned}
$$

Choosing $\omega=(3 t)^{1 / 3}$, and taking into account that $I_{1}+I_{2}=d_{k}(t)$, we easily arrive at

$$
d_{k}(t)=e^{i t k^{3}}
$$


This proves the analyticity of $\mathcal{T}_{\mathrm{K}}(\cdot)(\mathrm{f})(z)$, as function of $z \in \mathrm{D}_{\mathrm{R}}$. Finally, the relation $\mathcal{T}_{\mathrm{K}}(0)=\mathrm{I}$ is immediate in view of (21).

(ii) Let $|z| \leq r$. We obtain

$$
\begin{aligned}
\left|\mathcal{T}_{k}(t)(f)(z)-f(z)\right| & =\left|\sum_{k=0}^{\infty} a_{k} z^{k}\left[e^{i k^{3} t}-1\right]\right| \\
& =\left|\sum_{k=0}^{\infty} a_{k} z^{k}\left[-2 \sin ^{2}\left(k^{3} t / 2\right)+i \sin \left(k^{3} t / 2\right) \cos \left(k^{3} t / 2\right)\right]\right| \\
& \leq \sum_{k=0}^{\infty}\left|a_{k}\right| r^{k}\left|2 \sin ^{2}\left(k^{3} t / 2\right)\right|+\sum_{k=0}^{\infty}\left|a_{k}\right| r^{k}\left|2 \sin \left(k^{3} t / 2\right)\right| \\
& \leq \frac{t^{2}}{2} \sum_{k=0}^{\infty}\left|a_{k}\right| k^{6} r^{k}+|t| \sum_{k=0}^{\infty}\left|a_{k}\right| k^{3} r^{k},
\end{aligned}
$$

since $|\sin (x)| \leq|x|$, for all $x \in \mathbb{R}$.

(iii) We have

$$
\begin{aligned}
& \left|\mathcal{T}_{K}(t)(f)(z)-\mathcal{T}_{K}(s)(f)(z)\right| \\
& =\left|\sum_{k=0}^{\infty} a_{k} z^{k}\left[e^{i k^{3} t}-e^{i k^{3} s}\right]\right| \\
& =\sum_{k=0}^{\infty}\left[\cos \left(k^{3} t\right)-\cos \left(k^{3} s\right)+i\left(\sin \left(k^{3} t\right)-\sin \left(k^{3} s\right)\right)\right] \\
& \leq 4 \sum_{k=0}^{\infty}\left|a_{k}\right| r^{k}\left|\sin \left(\frac{k^{3}(t-s)}{2}\right)\right| \\
& \leq 2 \sum_{k=0}^{\infty}\left|a_{k}\right| k^{3} r^{k}|t-s| .
\end{aligned}
$$

(iv) From (i), it is immediate that $\mathcal{T}_{\mathcal{K}}(t+s)=\mathcal{T}_{K}(t) \mathcal{T}_{K}(s)$, for all $t, s \in \mathbb{R}_{+}$. The strong continuity of $\mathcal{T}_{K}(t)$ follows from (iii). We can argue as in the proof of Theorem 6.2.1 to deduce the first part of the statement in (iv). Let $f \in A^{*}\left(D_{R}\right)$. We can compute the generators of the semigroups $\mathcal{T}_{K}(t)$ and $\widetilde{\mathcal{T}}_{\mathrm{K}}(\mathrm{t}), \mathrm{t} \in \mathbb{R}_{+}$, respectively, as follows:

$$
\left(\frac{\mathrm{d}}{\mathrm{dt}} \mathcal{T}_{K}(\mathrm{t})(\mathrm{f})(z)\right)_{\mid \mathrm{t}=0}=i \sum_{k=0}^{\infty} k^{3} a_{k} e^{i k^{3} t} z^{k}=-\frac{\partial^{3}}{\partial \varphi^{3}} \mathcal{T}_{K}(\mathrm{t})(\mathrm{f})(z)
$$

and

$$
\left(\frac{\mathrm{d}}{\mathrm{dt}} \widetilde{\mathcal{T}}_{\mathrm{K}}(\mathrm{t})(\mathrm{f})(z)\right)_{\mid \mathrm{t}=0}=-i \sum_{k=0}^{\infty} k^{3} a_{k} e^{-i k^{3} t} z^{k}=\frac{\partial^{3}}{\partial \varphi^{3}} \frac{\mathrm{d}}{\mathrm{dt}} \widetilde{\mathcal{T}}_{\mathrm{K}}(\mathrm{t}) \mathrm{f}(z),
$$

for all $z=r e^{i \varphi} \in D_{R} \backslash\{0\}$. The proof of the theorem is complete. 
In what follows, the linearized Korteweg-de Vries equation from (5) is complexified by replacing $x \in \mathbb{R}$ with $z \in \Omega \subset \mathbb{C}$ directly in the equations. More precisely, we aim to study the following initial value problem

$$
\left\{\begin{array}{l}
\frac{\partial u}{\partial t}=\frac{\partial^{3} u}{\partial z^{3}}, \quad(t, z) \in \mathbb{R}_{+} \times \bar{\Omega}, \\
\lim _{t \backslash 0} u(t, z)=f(z), z \in \bar{\Omega} .
\end{array}\right.
$$

We look for classical solutions of (24) which belong to the class:

$$
u \in C^{1}\left(\mathbb{R}_{+} ; A^{\circ}\left(S_{r}\right)\right),
$$

where

$$
A^{\circ}\left(S_{r}\right):=\left\{f: \bar{S}_{r} \rightarrow \mathbb{C}: f \text { is analytic in } \bar{S}_{\mathrm{r}}\right\},
$$

i.e., $f$ is analytic in a domain that contains the closure $\bar{S}_{r}$ of

$$
S_{r}:=\{z=x+i y \in \mathbb{C}: x \in \mathbb{R},|y|<r\} .
$$

Theorem 3.2. Let $\mathrm{f} \in \mathrm{A}^{\circ}\left(S_{\mathrm{r}}\right)$ such that the following are satisfied:

(i) $\mathrm{f}^{\prime}$ is bounded on $\overline{\mathrm{S}}_{\mathrm{r}}$;

(ii) for all $|\mathrm{y}| \leq \mathrm{r}$, the integral $\mathrm{F}(\mathrm{s}, \mathrm{y})=\int_{-\infty}^{+\infty} \mathrm{e}^{-s \mathrm{x}} \mathrm{f}(\mathrm{x}+\mathrm{iy}) \mathrm{dx}$ is absolutely convergent for $\mathrm{s}=\mathrm{c}_{1}+\mathrm{i \tau}$;

(iii) for all $|\mathrm{y}| \leq \mathrm{r}$, the integral $\mathrm{G}(\mathrm{s}, \mathrm{y})=\int_{-\infty}^{+\infty} \mathrm{e}^{-s \mathrm{x}} \mathrm{f}^{\prime}(\mathrm{x}+\mathrm{iy}) \mathrm{dx}$ is absolutely convergent for $\mathrm{s}=\mathrm{c}_{1}+\mathrm{i} \tau$

(iv) for all $|\mathrm{y}| \leq \mathrm{r}, \int_{-\infty}^{+\infty}\left|\mathrm{F}\left(\mathrm{c}_{1}+\mathrm{i} \tau, \mathrm{y}\right)\right| \mathrm{d} \tau<+\infty$.

Setting

$$
\mathrm{T}_{\mathrm{KV}}(\mathrm{t})(\mathrm{f})(z):=\int_{-\infty}^{+\infty} \mathrm{K}(\mathrm{u}, \mathrm{t}) \mathrm{f}(z-\mathrm{u}) \mathrm{du},(\mathrm{t}, z) \in \mathbb{R}_{+} \times \overline{\mathrm{S}}_{\mathrm{r}},
$$

there holds $u(t)=T_{K V}(t)(f) \in C^{\infty}\left(\mathbb{R}_{+} ; A^{\circ}\left(S_{r}\right)\right)$, and $T_{K V}(t)(f)$ solves the initial value problem (24).

Proof. Let $f \in A^{\circ}\left(S_{r}\right)$ and decompose $f(z)=U(x, y)+i V(x, y)$, with $U$ and $V$ having continuous partial derivatives of first order. Moreover, $\mathrm{U}, \mathrm{V}$ satisfy the Cauchy-Riemann conditions at any $(x, y) \in \bar{S}_{r}$. We can write

$$
\begin{aligned}
T_{K V}(t)(f)(z) & =\int_{-\infty}^{+\infty} K(u, t) U(x-u, y) d u+i \int_{-\infty}^{+\infty} K(u, t) V(x-u, y) d u \\
& :=T_{1}(t, x, y)+i T_{2}(t, x, y) .
\end{aligned}
$$

Step 1. Let $z=x+i y \in \bar{S}_{r}$. Since

$$
f^{\prime}(z)=\frac{\partial u}{\partial x}(x, y)+i \frac{\partial V}{\partial x}(x, y),
$$


we note that

$$
f^{\prime \prime \prime}(z)=\frac{\partial^{3} u}{\partial x^{3}}(x, y)+i \frac{\partial^{3} V}{\partial x^{3}}(x, y) .
$$

We claim that for any fixed $|y| \leq r$, conditions (i)-(ii) in the statement of Theorem 7.1.1, are fulfilled for $T_{1}$ and $T_{2}$, with respect to $(t, x) \in \mathbb{R}_{+} \times \mathbb{R}$. As a consequence,

$$
\begin{aligned}
& \left\{\begin{array}{l}
\frac{\partial T_{1}}{\partial t}(t, x, y)=\frac{\partial^{3} T_{1}}{\partial x^{3}}(t, x, y), \quad(t, x, y) \in \mathbb{R}_{+} \times \bar{S}_{r} \\
\lim _{t \searrow 0} T_{1}(t, x, y)=U(x, y), \quad(x, y) \in \bar{S}_{r} .
\end{array}\right. \\
& \left\{\begin{array}{l}
\frac{\partial T_{2}}{\partial t}(t, x, y)=\frac{\partial^{3} T_{2}}{\partial x^{3}}(t, x, y),(t, x, y) \in \mathbb{R}_{+} \times \bar{S}_{r} \\
\lim _{t \searrow 0} T_{2}(t, x, y)=V(x, y),(x, y) \in \bar{S}_{r}
\end{array}\right.
\end{aligned}
$$

and, therefore, $\mathrm{T}_{\mathrm{KV}}(\mathrm{t})(\mathrm{f})$ solves (24). Indeed, by the first hypothesis above (see (i)), there exists $M>0$ such that

$$
\sup \left\{\left|f^{\prime}(z)\right|: z \in \bar{S}_{\mathrm{r}}\right\}=\left\|f^{\prime}\right\|_{\bar{S}_{\mathrm{r}}} \leq M .
$$

Now, from (26), $\frac{\partial u}{\partial x}(x, y)$ and $\frac{\partial V}{\partial x}(x, y)$ are bounded on $\bar{S}_{r}$. Therefore, for any fixed $|y| \leq r, u(x y)$ and $V(x, y)$ are continuous and of bounded variation with respect to $x \in \mathbb{R}$. Setting

$$
F_{1}(s, y)=\int_{-\infty}^{+\infty} e^{-s x} u(x, y) d x, F_{2}(s, y)=\int_{-\infty}^{+\infty} e^{-s x} V(x, y) d x
$$

clearly, $F(s, y)=F_{1}(s, y)+i F_{2}(s, y)$, and by virtue of (ii), both $F_{1}(s, y)$ and $F_{2}(s, y)$ are absolutely convergent, for a fixed (but otherwise arbitrary) $|y| \leq r$. Furthermore, in view of (iv), we deduce

$$
\int_{-\infty}^{+\infty}\left|F_{1}\left(c_{1}+i \tau, y\right)\right| d \tau<+\infty, \int_{-\infty}^{+\infty}\left|F_{2}\left(c_{1}+i \tau, y\right)\right| d \tau<+\infty .
$$

Therefore, we can apply Theorem 1.1 to the functions $T_{1}$ and $T_{2}$, respectively. This yields the above claim.

Step 2. Clearly, $\mathrm{T}_{\mathrm{KV}}(\mathrm{t})(\mathrm{f})$ belongs to the class (25) for any $\mathrm{f} \in \mathrm{A}^{\circ}\left(\mathrm{S}_{\mathrm{r}}\right)$. Indeed, the fact that $\partial_{x} T_{1}(\cdot, x, y)$ and $\partial_{x} T_{2}(\cdot, x, y)$ are continuous on $\bar{S}_{\mathrm{r}}$ was already proved in Step 1 . The existence and continuity of the partial derivatives $\partial_{y} T_{1}(\cdot, x, y), \partial_{y} T_{2}(\cdot, x, y)$ follow from condition (iii). Finally, the functions $T_{i}(\cdot, x, y), i=1,2$, also satisfy the Cauchy-Riemann equations since $U, V$ do. The proof is finished.

Remark 3.1. A simple example of boundary data in 24) that satisfies all the hypotheses of Theorem 3.2 is

$$
f(z)=e^{-z^{2}}
$$

In this case, one can prove that $\mathrm{F}(\mathrm{s}, \mathrm{y})=\sqrt{\pi} \mathrm{e}^{\mathrm{s}^{2} / 4} \cos (\mathrm{sy})$. 


\section{References}

[1] C. G. Gal, S. G. Gal and J. A. Goldstein, Evolution equations with real time variable and complex spatial variables, Complex Variables and Elliptic Equations, 53 (2008), No. 8, 753774.

[2] C. G. Gal, S. G. Gal and J. A. Goldstein, Higher order heat and Laplace type equations with real time variable and complex spatial variable, Complex Variables and Elliptic Equations, 55 (2010), No. 4, 357-373.

[3] C. G. Gal, S. G. Gal and J. A. Goldstein, Wave and telegraph equations with real time variable and complex spatial variables, Complex Variables and Elliptic Equations, 57 (2012), 91-109

[4] C. G. Gal, S. G. Gal and J. A. Goldstein, Schrödinger type equations with real time variable and complex spatial variables, Complex Variables and Elliptic Equations, 58 (2013), 415-430.

[5] O. Vallée and M. Suarez, Airy Functions and Applications to Physics, Imperial College Press, New Jersey, London, Singapore, 2004.

[6] K.V. Zhukovsky and G. Dattoli, Evolution of non-spreading Airy wavepackets in time dependent linear potentials, Applied Mathematics and Computation, 217(2011), 7966-7974.

[7] D.V. Widder, The airy transform, Amer. Math. Monthly, 86(1979), No. 4, 271-277. 